\title{
Measurement of adult human brain responses to breath hold- ing by multi-distance hyperspectral near-infrared spectroscopy
}

\author{
Zahida Guerouah ${ }^{1}$, Steve Lin ${ }^{2,3}$ and Vladislav Toronov 1,2,* \\ 1 Ryerson University, Faculty of Science, Department of Physics, 350 Victoria Street, Toronto, ON, Canada, \\ M5B 2K3; zguerouah@ryerson.ca \\ 2 Institute of Biomedical Engineering, Science and Technology (iBEST), Li Ka-Shing Knowledge Institute, 7th \\ Floor, LKS 735, 209 Victoria Street, Toronto, ON, M5B 1T8, Canada \\ 3 University of Toronto, Department of Medicine, 200 Elizabeth Street, Suite RFE 3-805, Toronto, ON, M5G \\ 2C4; Steve.Lin@unityhealth.to \\ * Correspondence: toronov@ryerson.ca
}

\begin{abstract}
A major limitation of near-infrared spectroscopy (NIRS) is its high sensitivity to the scalp and low sensitivity to the brain of adult humans. In the present work we use multi-distance hyperspectral NIRS (hNIRS) to investigate the optimal source-detector distances, range of wavelengths, and analysis techniques to separate cerebral responses to 30-s breath holds (BHs) from the responses in the superficial tissue layer in healthy adult humans. We observed significant responses to BHs in the scalp hemodynamics. Cerebral responses to BHs were detected in the cytochrome C oxidase redox $(\mathrm{rCCO})$ at $4 \mathrm{~cm}$ without using data from the short-distance channel. Using the data from the $1 \mathrm{~cm}$ channel in the two-layer regression algorithm showed that cerebral hemodynamic and $\mathrm{rCCO}$ responses also occurred at $3 \mathrm{~cm}$. We found that the waveband 700-900 $\mathrm{nm}$ was optimal for the detection of cerebral responses to $\mathrm{BH}$ in adults.
\end{abstract}

Keywords: near-infrared spectroscopy; brain; BOLD signal; breath holding; cytochrome C oxidase.

\section{Introduction}

Near-infrared spectroscopy (NIRS) has been proposed for human brain measurements in 1970s [1]. It also has been considered for clinical monitoring of cerebral status during various medical conditions in adults, such as cardiac surgeries, cardiac arrest, traumatic brain injury [2]. However, in such conditions significant circulatory and metabolic changes occur in the entire body, including the scalp, where NIRS sensitivity is maximal $[3,4]$. In general, high sensitivity to the scalp and low sensitivity to the brain of adult humans remains a main problem of NIRS in spite of numerous attempts to resolve it using different continuous-wave [5], time-domain [6], and frequency-domain approaches [7]. In particular, in several papers the combination of long and short source-detector channels was proposed and investigated [5, 8-12]. Other aspects of cerebral NIRS requiring investigation include optimization of the spectrum of wavelengths [13-15], and of the range of source-detector distances [14-16] for specific categories of subjects and patients: children, adults, and seniors. In the present work we approach the above aspects of cerebral NIRS using multi-distance hyperspectral NIRS (hNIRS). Apart from the ability to measure the optical properties of tissue at all NIR wavelengths simultaneously, hNIRS is the most robust technique to spectrally separate hemoglobin and redox cytochrome Coxidase (rCCO) changes $[1,17$. The latter is a direct marker of the cellular oxygen metabolism. In this work we used hNIRS to study the possibility to measure specific cerebral autoregulation changes concomitant with systemic changes at source-detector distances of $1 \mathrm{~cm}, 3 \mathrm{~cm}$, and $4 \mathrm{~cm}$ in healthy adults during breath holding $(\mathrm{BH})$ respiratory challenges. Since $\mathrm{BH}$ was proposed as a clinical paradigm to assess cerebral status in various clinical conditions such 
as stroke, concussion, etc. [18-20], the dynamics of cerebral responses to $\mathrm{BH}$ has been studied both by NIRS [21,22] and functional magnetic resonance imaging (fMRI) [23,24], which allows for a direct comparison of our results with many other studies.

\section{Materials and Methods}

\subsection{Measurement setup}

hNIRS custom sensor was placed on the left forehead over the Fp1 position according to the International 10-20 system [25] for the entire measurement procedure (see Fig. 1).

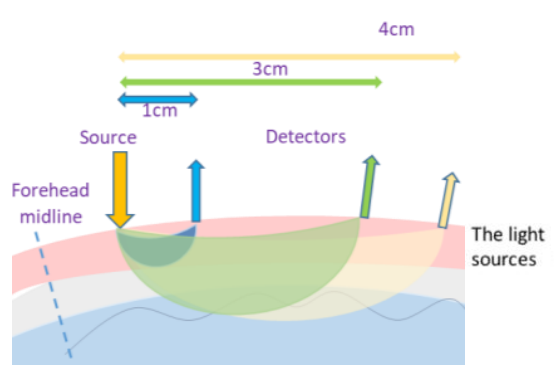

(a)

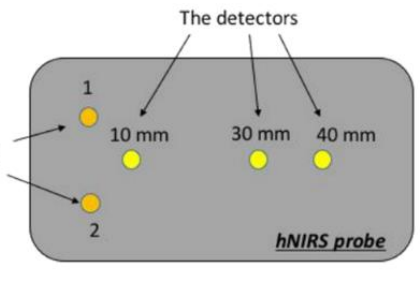

(b)

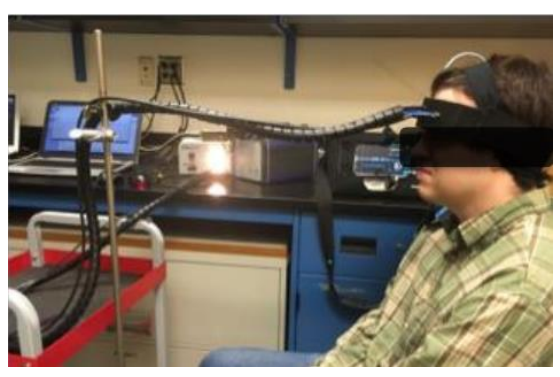

(c)

Figure 1. hNIRS measurement setup: a) approximate interrogated volumes in the scalp, skull, and brain; b) Optical sensor layout; c) Subject position.

The spectra were collected at the sampling rate of $2 \mathrm{~Hz}$ by three fiber optic spectrometers: AvaSpec (Avantes, CO), QE 65000 and USB 4000 (Ocean Optics, Dunedin, FL) at 4, 3 and $1 \mathrm{~cm}$, respectively, to separate the extracerebral and cerebral measurements. AvaSoft-Full software (Avantes, CO) was used to collect data from the AvaSpec spectrometer $(4 \mathrm{~cm})$, and The Spectra Suite (Ocean Optics, FL) software was used to collect the broadband continuous-wave hNIRS data from both Ocean Optics spectrometers with dark-signal correction. Data acquired at $1 \mathrm{~cm}$ represented the extra-cerebral layer (mostly scalp). Data acquired at $3 \mathrm{~cm}$ and $4 \mathrm{~cm}$ channels represented a combined extracerebral and cerebral tissue volume. The common spectral range of these spectrometers was from 650 to $1024 \mathrm{~nm}$. The spectrometers at $4 \mathrm{~cm}$ and $3 \mathrm{~cm}$ had a high signal-to-noise ratio (over 1000:1 single acquisition) and the slit width of $0.5 \mathrm{~mm}$ to provide sensitivity required to measure light at large distance from the source. Three custom-made 2-m-long optical fiber bundles (each made of seven $0.5 \mathrm{NA}, 400 \mu \mathrm{m}$ core diameter multimode polymer-clad fibers with broad UV/VIS/NIR spectral range of 400 to $2200 \mathrm{~nm}$ Thorlabs, NJ) connected spectrometers with the patient's head. Two other optical fiber bundles were used to connect the probe with a halogen lamp light source (Fiber-Lite Dc 950H Fiber Optic Illuminator, Dolan-Jenner, MA). The source light was injected into the tissue at two symmetric scalp locations (Fig. $1 \mathrm{~b}$ ) in order to increase the total light power without exceeding the maximum permissible exposure.

\subsection{Breath holding paradigm}

Data were obtained from 12 healthy adult participants (7 males, 5 females, 25-55 years old). All participants gave informed consent before participation and the experiment has been performed according to Ryerson University Research Ethics protocol. Participants were audibly cued to perform a 30 -second $\mathrm{BH}$ at the end of expiration. $\mathrm{BH}$ was repeated three times (60-90 s, 180-210 s, and 300-330 s from the beginning of the recording) with 90-second rest intervals. Long time intervals between BHs were used to avoid resonance induction of systemic Mayer waves in arterial blood pressure [26,27]. All data sets including the baseline periods before and after BHs were acquired during 10 minutes.

\subsection{Data processing}

All our custom signal processing methods were implemented in MATLAB (Mathworks, MA, Version R2020b). At the pre-processing stage the data from all spectrometers 
were resampled in both time- and spectral- domains to the time step of $0.5 \mathrm{~s}$ and wavelength step of $1 \mathrm{~nm}$ between 650 and $1024 \mathrm{~nm}$, filtered in the time domain using a bandpass filter with the window of $0.01-0.3 \mathrm{~Hz}$, and smoothed in the spectral domain with the median filter of the $20 \mathrm{~nm}$ width. To analyze the chromophores, we used two custom data processing approaches. Our first algorithm was based on the analytical solution to the diffusion equation12-13 for the semi-infinite homogeneous medium. It allows for measuring the bulk absolute concentrations of tissue $\mathrm{HbO} 2$ and $\mathrm{HHb}$, and changes in rCCO concentration without accounting for the layered tissue structure. The baseline concentrations of $\mathrm{HbO} 2$ and $\mathrm{HHb}$ were calculated by performing a non-linear least square fitting 10 of the measured absorbance spectrum at each moment of time by the analytical solution to the diffusion equation, in which the optical absorption coefficient $\mu_{a}(\lambda)$ was modeled as

$$
\mu_{a}(\lambda)=[H b] \varepsilon(\lambda)_{H b}+\left[H_{b} O_{2}\right] \varepsilon(\lambda)_{H_{b O}}
$$

according to Beer-Lambert law, and the reduced scattering coefficient $\mu_{s}{ }^{\prime}(\lambda)$ as a function of wavelength $\lambda$ was modeled using the power law as described in [28]. In Eq. (1) and below the square brackets denote concentrations measured in micromolars $(\mu \mathrm{M})$. The temporal changes in the hemoglobin concentrations $\mathrm{HbO} 2, \mathrm{Hb}$ and $\mathrm{rCOO}$ were resolved using a two-step data-fitting algorithm12,15 (also based on the same analytical solution to the diffusion equation) by relating changes in $\mathrm{HbO} 2, \mathrm{Hb}$ and $\mathrm{rCOO}$ to the changes in the optical absorbance as

$\Delta \mu_{a}(\lambda, t)=\Delta[H b](t) \varepsilon(\lambda)_{H b}+\Delta\left[H b O_{2}\right](t) \varepsilon(\lambda)_{H_{b O}}+\Delta[C C O](t) \varepsilon(\lambda)_{c c o}$

where $\varepsilon(\lambda)_{x}$ were the spectra of the extinction coefficients of $\mathrm{HbO} 2, \mathrm{Hb}$, and $\mathrm{rCOO} 28$ 29.

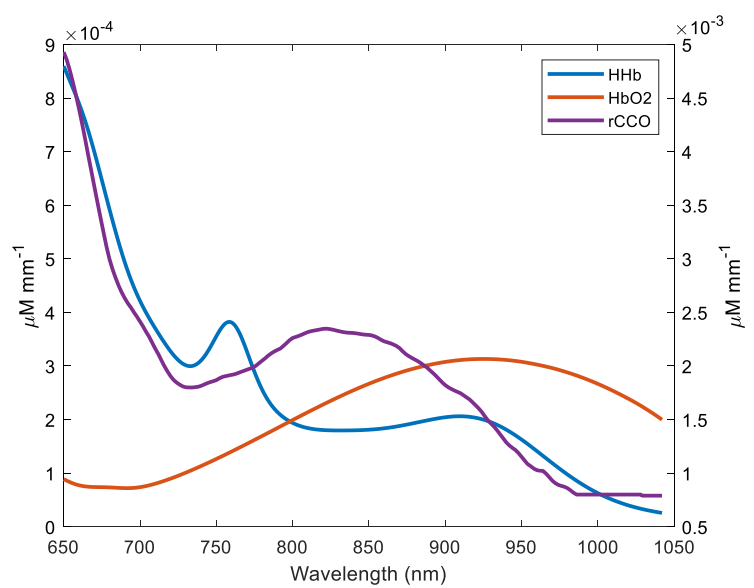

Figure 2. Specific absorption spectra of $\mathrm{HHb}, \mathrm{HbO} 2$, and $\mathrm{rCCO}$.

The data fitting was performed is 2 steps: first $\Delta[\mathrm{HbO} 2]$ and $\Delta[\mathrm{HHb}]$ were calculated assuming $\Delta[\mathrm{CCO}]=0$, and after that $\Delta[\mathrm{CCO}]$ was calculated and retained non-zero only if the addition of $\Delta[\mathrm{CCO}]$ resulted in the improvement of the fit quality of at least $20 \%$ in terms of the norm of residuals. Additional details on recovering the absolute values and changes of chromophore concentrations from the hNIRS data could be found in $[28,29]$.

As the fraction of oxygenated hemoglobin relative to the total hemoglobin in the blood, the cerebral tissue saturation of oxygen was calculated as:

$$
t \mathrm{SO}_{2}=\frac{\left[\mathrm{HbO}_{2}\right]}{\left[\mathrm{HbO}_{2}\right]+[\mathrm{Hb}]}(\%)
$$


In order to assess differences between the cerebral and scalp responses to $\mathrm{BH}$ we used another common NIRS model: the modified Lambert-Beer law for a two-layer medium [30,31]. This model assumes that at any moment of time $t$ the absorbance measured at $1 \mathrm{~cm}$ and at the wavelength $\lambda$ could be related to the changes of the absorption coefficient of the scalp only $\Delta \mu_{a, s}(\lambda, t)$ :

$$
\Delta O D_{1 \mathrm{~cm}}(\lambda, t)=L_{s 1 \mathrm{~cm}} \Delta \mu_{a, s}(\lambda, t)
$$

where $L_{s 1 \mathrm{~cm}}$ is the optical pathlength in the scalp at $1 \mathrm{~cm}$, and the absorbance at $3 \mathrm{~cm}$ and $4 \mathrm{~cm}$ could be expressed as

$$
\begin{aligned}
& \Delta O D_{3 c m}(\lambda, t)=L_{s 3 c m} \Delta \mu_{a, s}(\lambda, t)+L_{c 3 c m} \Delta \mu_{a, c}(\lambda, t) \\
& \Delta O D_{4 c m}(\lambda, t)=L_{s 4 c m} \Delta \mu_{a, s}(\lambda, t)+L_{c 4 c m} \Delta \mu_{a, c}(\lambda, t)
\end{aligned}
$$

where $\Delta \mu_{a, c}(\lambda, t)$ is the change in the cerebral absorption coefficient, $L_{s} 3 \mathrm{~cm}$ and $L_{s} 4 \mathrm{~cm}$ are the scalp partial pathlength at $3 \mathrm{~cm}$ and $4 \mathrm{~cm}$, respectively, and $L_{c 3} 3 \mathrm{~cm}$ and $L_{c} 4 \mathrm{~cm}$ are the cerebral partial pathlength at $3 \mathrm{~cm}$ and $4 \mathrm{~cm}$, respectively. Since the pathlengths in equations (4-6) were specific for every individual and unknown, $\Delta \mu_{a, s}$ could not be algebraically excluded to find $\Delta \mu_{a, c}$. However, since equations (4)-(5) were linear with respect to the common time-dependent $\Delta \mu_{a, s}(\lambda, t)$ and $\Delta \mu_{a, c}(\lambda, t)$, for every wavelength $\lambda$ one could exclude the time course common with $\Delta \mu_{a, s}(\lambda, t)$ from $\Delta O D_{3 \mathrm{~cm}}(\lambda, t)$ and $\Delta O D_{4 c m}(\lambda, t)$. Indeed, one could rewrite Eqs. (5) and (6) in the form

$$
\begin{aligned}
& \Delta O D_{3 c m}(\lambda, t)=L_{c 3 c m} \delta \mu_{a, c}(\lambda, t)+\left(L_{c 3 c m}+L_{s 3 c m}\right)\left(L_{(s 1 c m)}\right)^{-1} \Delta O D_{1 c m}(\lambda, t) \\
& \Delta O D_{4 c m}(\lambda, t)=L_{c 4 c m} \delta \mu_{a, c}(\lambda, t)+\left(L_{c 4 c m}+L_{s 4 c m}\right)\left(L_{(s 1 c m)}\right)^{-1} \Delta O D_{1 c m}(\lambda, t)
\end{aligned}
$$

where

$$
\delta \mu_{a, c}(\lambda, t)=\Delta \mu_{a, c}(\lambda, t)-\Delta \mu_{a, s}(\lambda, t)
$$

represents the difference between the cerebral and scalp responses to BH. Note that Eqs. (7) and (8) are linear with respect $\Delta O D_{1 \mathrm{~cm}}(\lambda, t)$ and $\delta \mu_{a, c}(\lambda, t)$, and also we expect that the latter functions of time and wavelength be uncorrelated in the time domain. Therefore the time-domain linear regression coefficients between $\Delta O D_{1 \mathrm{~cm}}(\lambda, t)$ and $\triangle O D_{3 \mathrm{~cm}}(\lambda, t)$ and between $\Delta O D_{1 \mathrm{~cm}}(\lambda, t)$ and $\Delta O D_{4 \mathrm{~cm}}(\lambda, t)$ should be close to $\beta_{1,3 \mathrm{~cm}} \approx\left(L_{c 3 \mathrm{~cm}}+\right.$ $\left.L_{s 3 \mathrm{~cm}}\right)\left(L_{\left(s_{11 \mathrm{~cm}}\right)}\right)^{-1}$ and $\beta_{1,4 \mathrm{~cm}} \approx\left(L_{c 4 \mathrm{~cm}}+L_{s 4 \mathrm{~cm}}\right)\left(L_{\left(s_{11 \mathrm{~cm}}\right)}\right)^{-1}$, respectively. Then, by subtracting $\beta_{1,3 \mathrm{~cm}}(\lambda) \Delta O D_{1 \mathrm{~cm}}(\lambda, t)$ and $\beta_{1,4 \mathrm{~cm}}(\lambda) \Delta O D_{1 \mathrm{~cm}}(\lambda, t)$ from Eqs. (7) and (8) we obtain:

$$
\delta O D_{3 \mathrm{~cm}}(\lambda, t) \approx L_{c 3 \mathrm{~cm}} \delta \mu_{a, c}(\lambda, t), \text { and } \delta O D_{4 \mathrm{~cm}}(\lambda, t) \approx L_{c 4 c m} \delta \mu_{a, c}(\lambda, t)
$$

Note that at all wavelengths the true cerebral response to $\mathrm{BH}$ could have a component close to the scalp response. Therefore, $\delta O D_{3,4} \mathrm{~cm}(\lambda, t)$ represent not the pure time course of the cerebral absorption coefficient, but rather the difference between the cerebral and scalp absorption time courses.

In accordance with Eq. (2) one could model $\delta O D_{3,4} \mathrm{~cm}(\lambda, t)$ as a linear combinations of the contributions from $\Delta[\mathrm{Hb}]_{c, s}(t), \Delta\left[\mathrm{HbO}_{2}\right]_{c, s}(t)$, and $\Delta[\mathrm{CCO}]_{c, s}(t)$ :

$$
\delta O D_{3,4 \mathrm{~cm}}(\lambda, t) \sim \delta[\mathrm{Hb}]_{c, s}(t) \varepsilon(\lambda)_{\mathrm{Hb}}+\delta\left[\mathrm{HbO}_{2}\right]_{c, s}(t) \varepsilon(\lambda)_{\mathrm{HbO}_{2}}+\delta[\mathrm{CCO}]_{c, s}(t) \varepsilon(\lambda)_{r c c o}
$$

By applying the stepwise linear regression spectral unmixing to the linear model (9) (where the regressors are the extinction coefficients $\left.\varepsilon(\lambda)_{x}\right)$ one could obtain $\delta[H b]_{c, s}(t)$, $\delta\left[\mathrm{HbO}_{2}\right]_{c, s}(t)$, and $\delta[\mathrm{CCO}]_{c, s}(t)$, whose time courses represent the differences between the cerebral and scalp responses in $\mathrm{HHb}, \mathrm{HbO} 2$, and $\mathrm{rCCO}$, respectively. In this spectral 
unmixing step the initial regression model did not include $\delta[C C O]_{c, s}(t)$. It was added to the model at the second step of the stepwise regression only if the $p$-value corresponding to non-zero $\delta[C C O]_{c, s}(t)$ was greater than 0.05 .

In addition, we also used Eqs. (10) to calculate $\delta[t H b]_{c, s}$ to calculate differential response in the total hemoglobin. $\delta[t H b]_{c, s}$ was calculated not using the spectral unmixing, but directly using Eqs. (10) around $800 \mathrm{~nm}$ since at this wavelength $\varepsilon_{\mathrm{Hb}}=\varepsilon_{\mathrm{HbO}_{2}}$. Since near $800 \mathrm{~nm} \varepsilon(\lambda)_{r c c o}$ is approximately 10 time greater than $\varepsilon_{H b}, \delta[t H b]_{c, s}$ could include a crosstalk with $\delta[r C C O]_{c, s}(t)$.

Since the values of cerebral pathlengths $L_{c 3 \mathrm{~cm}}$ and $L_{c 4 \mathrm{~cm}}$ were unknown, for the quantitative comparison in $\mu \mathrm{M}$ with the responses obtained using the homogeneous tissue model (explained above) we normalized $\Delta[\mathrm{Hb}]_{c, s}(t), \Delta\left[\mathrm{HbO}_{2}\right]_{c, s}(t)$, and $\Delta[\mathrm{rCCO}]_{c, s}(t)$ to the pathlength corresponding to the homogeneous tissue equal to the product of the differential pathlength factor (known be close to 6 for mid-age human forehead) and the source-detector distance.

\section{Results}

Figure 3 shows the assessment of de-noised signals in all three channels and at all wavelengths in terms of the cross-subject averaged temporal correlation (first and second rows), temporal logarithmic signal means (third row, used for the data quality assessment), and temporal standard deviations (fourth row).

Since the noise was filtered out, the latter represent the average magnitude of changes at different wavelengths. The correlation coefficient maps (first row) show that signals at all wavelengths from $700 \mathrm{~nm}$ to over $900 \mathrm{~nm}$ and in all channels were positively correlated. The latter means that there were no pairs of signals at any wavelengths from $700 \mathrm{~nm}$ to $900 \mathrm{~nm}$ and in any channels where temporal changes occurred simultaneously in opposite directions. Also from the correlation maps and same-wavelength correlation plots (second row) one could see that all signals between $750 \mathrm{~nm}$ and $900 \mathrm{~nm}$ were highly correlated (correlation coefficient $>0.6$ ). Correlations were low for the wavelengths longer than 900 $\mathrm{nm}$ in the $4-\mathrm{cm}$ channel. While the mean signal value in the $4-\mathrm{cm}$ channel at these wavelengths was high (third row), the amplitude of changes (fourth row) was small. This lack of changes should be due to fact that at the $4 \mathrm{~cm}$ source-detector separation the light of wavelengths greater than $900 \mathrm{~nm}$ mostly interrogated the volume which belongs to the skull where no changes occurred. For the above reasons the further analysis was performed using the waveband from $750 \mathrm{~nm}$ to $900 \mathrm{~nm}$.

Figure 4 shows the cross-subject averaged changes in [HbO2], [HHb], [rCCO], and [rSO2] measured at $1 \mathrm{~cm}, 3 \mathrm{~cm}$, and $4 \mathrm{~cm}$ using the homogeneous tissue model and 750 $900 \mathrm{~nm}$ waveband. One could see that the hemoglobin signals showed responses to BHs in all channels, while rCCO exhibited clear responses only at $4 \mathrm{~cm}$. Table 1 presents the average amplitude and temporal feature values characteristic for the chromophore responses to BHs.

The time courses of $\mathrm{HbO} 2$ responses appeared similar in all channels except at $4 \mathrm{~cm}$, where $\mathrm{HbO} 2$ responses to the 2 nd and 3rd trials showed 10-15 s delays. The latter could be an indication of a cerebral response predominance in the $4 \mathrm{~cm}$ channel (see the Discussion section below). However, $\mathrm{HbO} 2$ signal at $3 \mathrm{~cm}$ was very similar to the $1-\mathrm{cm}$ signal. 

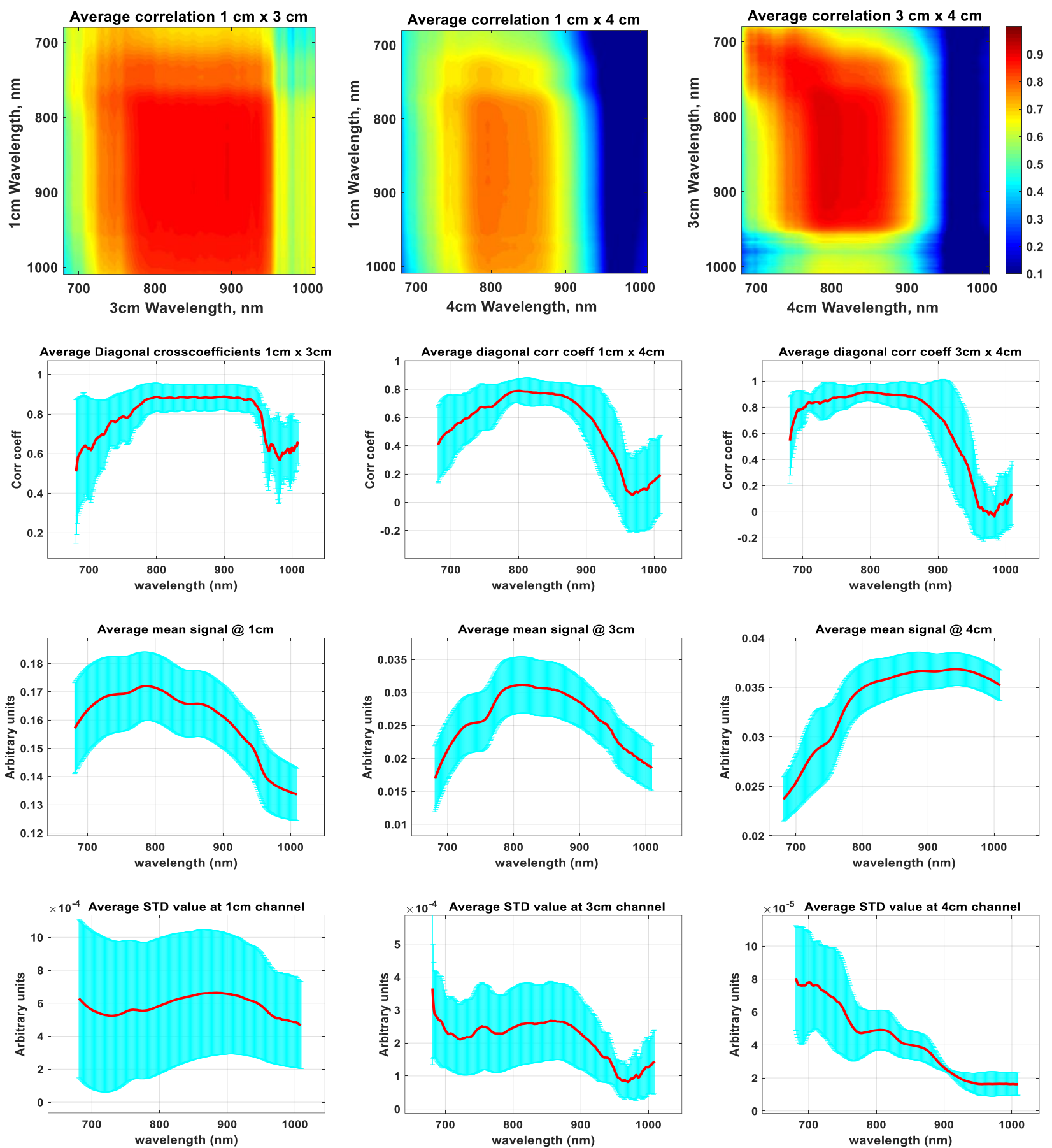

Figure 3. Hyperspectral signal analysis: cross-subject averaged correlation, temporal means, and temporal standard deviations for signals from $1 \mathrm{~cm}, 3 \mathrm{~cm}$, and $4 \mathrm{~cm}$ channels. Blue color shows the cross-subject standard deviations.

The common features of $\mathrm{HHb}$ responses to BHs in all channels were the initial dips followed by quick increases peaking up 4-10 s after the end of BH. The depth of initial dips was much greater in the $1 \mathrm{~cm}$ channel than in $3 \mathrm{~cm}$ and $4 \mathrm{~cm}$ channels (see Table 1). The $\mathrm{HHb}$ peak time was longest for the $1 \mathrm{~cm}$ channel (about $10 \mathrm{~s}$, see Table 1). Much sharper peaks and faster falls in the $\mathrm{HHb}$ responses at $4 \mathrm{~cm}$ compared to those features at $1 \mathrm{~cm}$ and $3 \mathrm{~cm}$ might indicate a prevalence of the cerebral component in the $4-\mathrm{cm} \mathrm{HHb}$ responses. In particular, the faster falls of $\mathrm{HHb}$ at $4 \mathrm{~cm}$ than at $1 \mathrm{~cm}$ and $3 \mathrm{~cm}$ could be due to the cerebral autoregulation restoring the oxygen concentration in the brain faster than in extracerebral tissues. At $3 \mathrm{~cm}$ the $\mathrm{HHb}$ response shows features of both $1-\mathrm{cm}$ and $4 \mathrm{~cm}$ responses. 

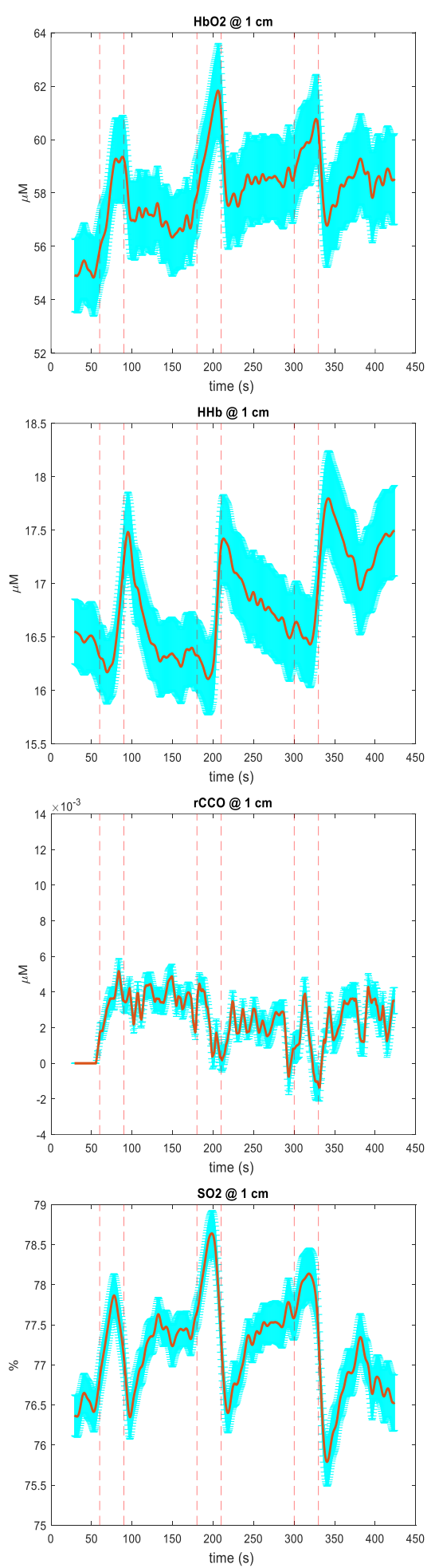
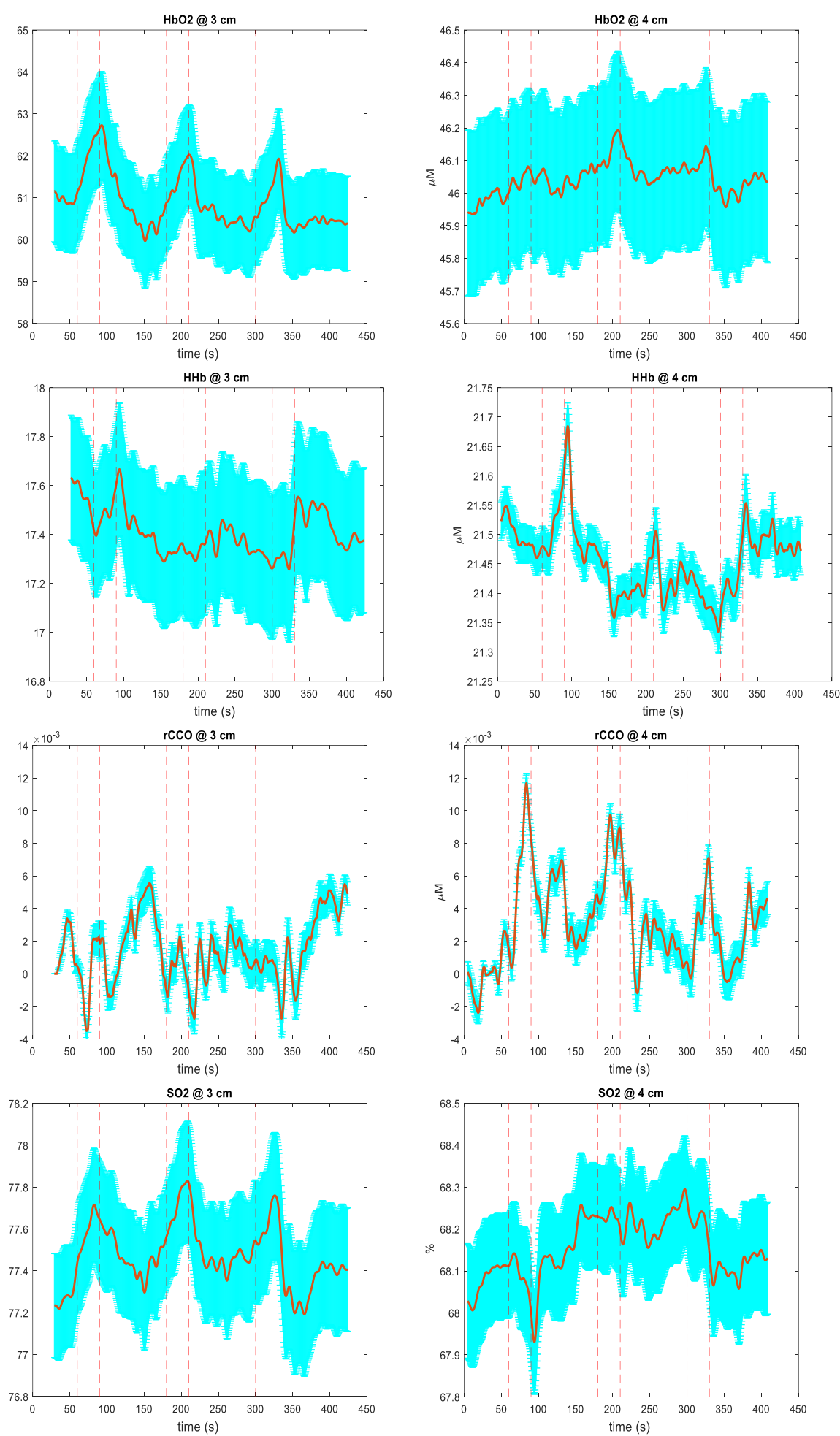

Figure 4. Cross-subject averaged changes in [HbO2], [HHb], [rCCO], and regional $\mathrm{SO} 2$ measured at $1 \mathrm{~cm}, 3 \mathrm{~cm}$, and $4 \mathrm{~cm}$ using the homogeneous tissue model and 750-900nm waveband. Blue color shows the cross-subject standard deviations.

rCCO signals from 1-cm and 3-cm channels show no clear responses to BHs. At $4 \mathrm{~cm}$ the rCCO responses were very clear, which also might indicate that these responses belong solely to the cerebral tissue. Each rCCO fall after the end of $\mathrm{BH}$ is quickly followed by a rebound which also could be a result of the faster restoration of the oxygen supply in the brain than in other tissues. Note that rCCO peak changes had about 10 times smaller magnitudes than $\mathrm{HbO} 2$ and $\mathrm{HHb}$ changes (Table 1), which was in agreement with the fact 
that the concentration of CCO in the brain is approximately 10 times smaller than the concentration of hemoglobin.

In Fig. 4 the SO2 time course at $4 \mathrm{~cm}$ was also very different from those at $1 \mathrm{~cm}$ and $3 \mathrm{~cm}$, which were very similar to each other by showing SO2 increases characteristic for the scalp tissue. However, the quick SO2 falls at $4 \mathrm{~cm}$ in the end of each $\mathrm{BH}$ should not be interpreted as the failure of cerebral autoregulation but rather these falls result from the smaller magnitude in the $\mathrm{HbO} 2$ increase than in the $\mathrm{HHb}$ increase due to the difference in the partial volume of the skull interrogated by light at shorter and longer wavelengths.

Table 1. Cross-subject average peak and dip magnitudes and times. For $\mathrm{HbO} 2$ and $\mathrm{rCCO}$ the peak magnitudes were measured from the 20-s averaged values before BHs. The dip magnitudes correspond to the difference between the peak and the next dip (see

Fig.4). Since for $\mathrm{HHb}$ the dips occurred before peaks, the dip magnitudes were measured from the 20-s averaged values before

$\mathrm{BHs}$, and the peak magnitudes were measured from the preceding dips. The peak and dip times were measured from the beginning of BHs.

\begin{tabular}{|c|c|c|c|c|c|c|c|}
\hline \multicolumn{8}{|c|}{$\mathrm{HbO} 2$} \\
\hline & & \multicolumn{2}{|c|}{$1^{\text {st }} \mathrm{BH}$} & \multicolumn{2}{|c|}{$2^{\text {nd }} \mathrm{BH}$} & \multicolumn{2}{|c|}{$3^{\text {rd }} \mathrm{BH}$} \\
\hline & & Peak & Dip & Peak & Dip & Peak & Dip \\
\hline \multirow[t]{2}{*}{$1 \mathrm{~cm}$} & $\Delta(\mu \mathrm{M})$ & $5.7 \pm 1.4$ & $-5.4 \pm 0.8$ & $6.3 \pm 1.5$ & $-7.3 \pm 1.2$ & $3.7 \pm 0.9$ & $-6.8 \pm 1.3$ \\
\hline & $\mathrm{t}(\mathrm{s})$ & $25.4 \pm 2.5$ & $47.2 \pm 2.1$ & $24.6 \pm 1.7$ & $45.9 \pm 2.0$ & $23.8 \pm 2.3$ & $44.4 \pm 1.8$ \\
\hline \multirow[t]{2}{*}{$3 \mathrm{~cm}$} & $\Delta(\mu \mathrm{M})$ & $2.8 \pm 0.6$ & $-3.1 \pm 0.7$ & $2.86 \pm 0.64$ & $-3.1 \pm 0.6$ & $2.1 \pm 0.5$ & $-3.0 \pm 0.6$ \\
\hline & $\mathrm{t}(\mathrm{s})$ & $33.6 \pm 1.2$ & $53.9 \pm 3.5$ & $29.6 \pm 1.3$ & $47.2 \pm 2.5$ & $31.6 \pm 1.3$ & $46.1 \pm 1.6$ \\
\hline \multirow[t]{2}{*}{$4 \mathrm{~cm}$} & $\Delta(\mu \mathrm{M})$ & $0.2 \pm 0.0$ & $-0.4 \pm 0.1$ & $0.2 \pm 0.1$ & $-0.4 \pm 0.1$ & $0.2 \pm 0.1$ & $-0.4 \pm 0.1$ \\
\hline & $\mathrm{t}(\mathrm{s})$ & $28.0 \pm 2.4$ & $40.2 \pm 3.3$ & $28.8 \pm 2.1$ & $43.7 \pm 3.4$ & $26.6 \pm 2.1$ & $46.0 \pm 3.2$ \\
\hline \multicolumn{8}{|c|}{$\mathrm{rCCO}$} \\
\hline & & Peak & Dip & Peak & Dip & Peak & Dip \\
\hline \multirow[t]{2}{*}{$4 \mathrm{~cm}$} & $\Delta^{*} 10^{-2}$ & $1.1 \pm 0.2$ & $-1.5 \pm 0.4$ & $0.9 \pm 0.1$ & $-1.6 \pm 0.3$ & $0.8 \pm 0.2$ & $-1.3 \pm 0.2$ \\
\hline & $\mathrm{t}(\mathrm{s})$ & $23.4 \pm 1.86$ & $43.52 \pm 1.98$ & $21.42 \pm 1.41$ & $40.51 \pm 2.07$ & $24.6 \pm 1.78$ & $41.69 \pm 1.71$ \\
\hline \multicolumn{8}{|c|}{$\mathrm{HHb}$} \\
\hline & & Peak & Dip & Peak & Dip & Peak & Dip \\
\hline \multirow[t]{2}{*}{$1 \mathrm{~cm}$} & $\Delta(\mu \mathrm{M})$ & $1.9 \pm 0.4$ & $-0.7 \pm 0.3$ & $2.1 \pm 0.4$ & $-0.6 \pm 0.2$ & $1.9 \pm 0.3$ & $-0.5 \pm 0.2$ \\
\hline & $t(s)$ & $37.1 \pm 1.9$ & $14.0 \pm 2.2$ & $39.6 \pm 2.9$ & $14.5 \pm 2.2$ & $40.8 \pm 2.0$ & $15.5 \pm 2.0$ \\
\hline \multirow[t]{2}{*}{$3 \mathrm{~cm}$} & $\Delta(\mu \mathrm{M})$ & $0.7 \pm 0.3$ & $-0.1 \pm 0.6$ & $0.6 \pm 0.2$ & $-0.1 \pm 0.1$ & $0.6 \pm 0.2$ & $-0.1 \pm 0.2$ \\
\hline & $t(s)$ & $37.9 \pm 1.6$ & $15.9 \pm 1.98$ & $37.5 \pm 1.4$ & $18.0 \pm 2.4$ & $40.3 \pm 2.6$ & $19.7 \pm 1.8$ \\
\hline \multirow[t]{2}{*}{$4 \mathrm{~cm}$} & $\Delta(\mu \mathrm{M})$ & $0.4 \pm 0.1$ & $-0.01 \pm 0.03$ & $0.31 \pm 0.05$ & $-0.01 \pm 0.03$ & $0.36 \pm 0.09$ & $-0.01 \pm 0.03$ \\
\hline & $\mathrm{t}(\mathrm{s})$ & $34.7 \pm 0.7$ & $14.4 \pm 1.6$ & $34.8 \pm 0.7$ & $18.2 \pm 2.1$ & $34.1 \pm 1.3$ & $15.9 \pm 1.8$ \\
\hline
\end{tabular}

As explained above, in order to reveal differences between responses to BHs measured in different channels we performed the time-spectral linear regression analysis. For the regression analysis the range of wavelengths was limited to 750-900 $\mathrm{nm}$ where the highest correlation between different channels was detected (see Fig.3). Figure 5 shows the cross-subject average correlation and standard deviations spectra of signals from the $3-\mathrm{cm}$ and $4-\mathrm{cm}$ channels after regressing out changes measured the $1-\mathrm{cm}$ channel. One could see that the correlation coefficient for all wavelengths in both channels was greater than 0.6 between $750 \mathrm{~nm}$ and $900 \mathrm{~nm}$. The correlation coefficient for the same wavelengths in both channels (corresponding to the diagonal of the correlation map in Fig. 5) was particularly high for all wavelengths. This high correlation indicated that after regressing out the superficial changes, both $3-\mathrm{cm}$ and $4-\mathrm{cm}$ channels exhibited very similar time courses 
at all wavelengths. Also in Fig. 5 one can see that the spectra of average amplitudes of changes (measured by SDs) were similar in shape to those before regression (shown in Fig. 3). They showed peaks at the $\mathrm{HHb}$ and $\mathrm{rCCO}$ absorption maxima around $760 \mathrm{~nm}$ and $830 \mathrm{~nm}$, respectively (compare with Fig. 2), and decrease at wavelengths longer than 870 $\mathrm{nm}$, corresponding to maximum $\mathrm{HbO} 2$ absorption. Therefore, after the regression the $\mathrm{HbO} 2$ changes should have smaller magnitudes than the $\mathrm{HHb}$ and $\mathrm{rCCO}$ responses.
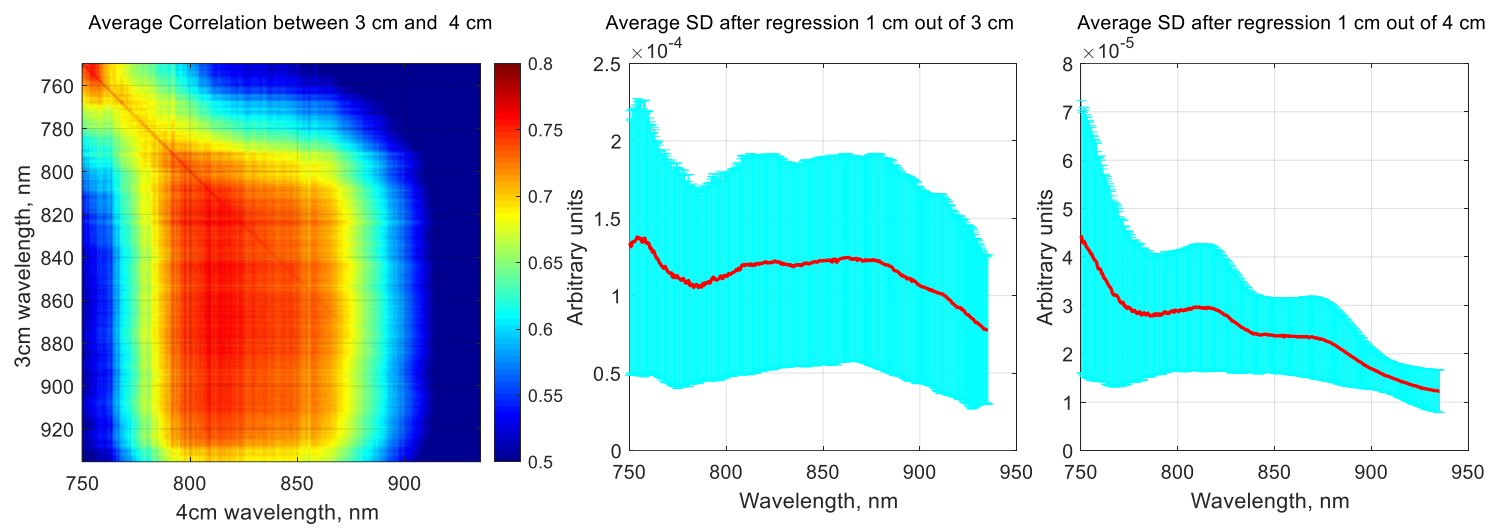

Figure 5. Cross-subject average correlation and standard deviations spectra of signals from the 3-cm and 4-cm channels after regressing out changes measured at the $1-\mathrm{cm}$ channel.

In Figure 6 the green and blue curves show the cross-subject averaged changes in [HbO2], [HHb], [tHB], and [rCCO] calculated by applying the spectral-domain regression to the residuals after regressing out the in time-domain the changes measured at the $1-\mathrm{cm}$ channel from the signals measured at the $3-\mathrm{cm}$ and $4-\mathrm{cm}$ channels, respectively. The [tHB] changes were computed by averaging changes in the $5 \mathrm{~nm}$ waveband around $800 \mathrm{~nm}$ where the $\mathrm{HHb}$ and $\mathrm{HbO} 2$ specific absorptions were equal. Note that the changes in Fig. 6 show the differences in the time courses of changes in the deep and superficial tissues. (Note that signals measured at different channels never change in opposite directions, which follows from the correlation analysis shown in Fig. 3 and explained above.) In particular, the rising slopes seen on the curves in Fig. 6 correspond to the time intervals when changes measured at 3 or $4 \mathrm{~cm}$ increase faster than changes measured at $1 \mathrm{~cm}$. Conversely, the curves in Fig. 6 show negative slopes during the times when changes measured at long-distance channels decrease faster than changes measured at the $1-\mathrm{cm}$ channel. The fact that the $\mathrm{HbO} 2$ responses to $\mathrm{BHs}$ are not very clear means that the time courses of [HbO2] changes in the superficial and cerebral tissues are similar. (The "noise" seen if Fig. 6 was mostly due to the respiratory sinus arrhythmia between BHs). In Fig. 6 one can see that the peak times of [HHb], [tHB], and [rCCO] responses during all three $\mathrm{BH}$ episodes were between 15 and 25 seconds after the BH onsets, which was similar to the peak times of rCCO responses shown in Fig. 4, but very different from the peak times of $\mathrm{HbO} 2$ and $\mathrm{HHb}$ responses shown in Fig. 4, which never occur earlier than $26 \mathrm{~s}$ from the beginning of a $\mathrm{BH}$ episode (see also Table 1). Some details in the $\mathrm{HHb}, \mathrm{tHB}$, and $\mathrm{rCCO}$ responses in Fig. 6 were quite different. In particular, during $\mathrm{BH}$ episodes the $\mathrm{HHb}$ curves showed initial dips during the first 10-15 s turning into the fast increases after that. During the same initial periods of $\mathrm{BHs}$ the $\mathrm{tHB}$ curves show steady positive changes, while rCCO curves show almost no changes during the first $10 \mathrm{~s}$. These differences indicated that the responses to BHs in Fig. 6 indeed corresponded to different chromophores and did not result from a crosstalk between different NIR wavelengths. The fact that in Fig. 6 the curves corresponding to the 4-cm channel showed similar changes to the curves corresponding to the 3-cm channel but with much smaller magnitudes is due to the overall smaller magnitudes of changes in the $4 \mathrm{~cm}$ channel as shown in Fig. 3 and explained above. 

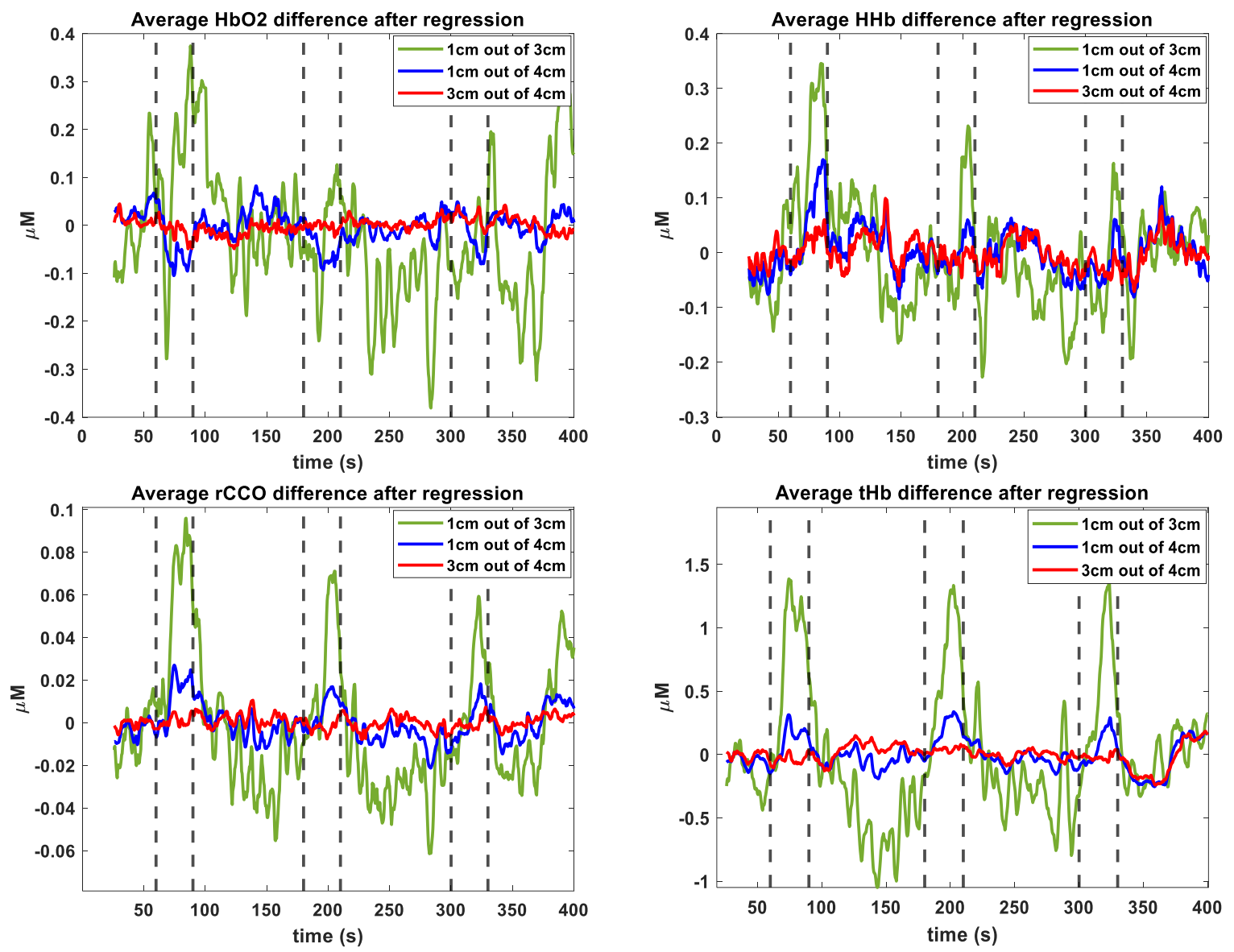

Figure 6. Cross-subject averaged changes in [HbO2], [HHb], [tHB], and [rCCO] calculated from the time-domain regression residuals using stepwise linear regression in spectral domain.

In addition, the red curves in Fig. 6 correspond to regressing out the $3-\mathrm{cm}$ changes from the $4-\mathrm{cm}$ changes. The fact that these curves show almost no changes means that after regressing out the changes due to the contribution from the superficial tissues the time courses of changes at all wavelengths in both $3-\mathrm{cm}$ and $4-\mathrm{cm}$ channels were very similar, which was also in agreement with the high correlation between signals from these channels at all wavelengths as shown in Fig. 5.

\section{Discussion}

Our results showed that BHs induced significant hemodynamic responses in all three channels including the $1 \mathrm{~cm}$ channel which interrogated only the scalp and skull. Hemodynamic responses measured by the $3-\mathrm{cm}$ channel were quite similar to those measured at $1 \mathrm{~cm}$. In Fig. 4, only signals measured at $4 \mathrm{~cm}$ showed different responses than the extracerebral tissue. The clearest difference from the scalp response was observed at $4 \mathrm{~cm}$ in the $\mathrm{HHb}$ and $\mathrm{rCCO}$ responses (Fig. 4). No rCCO responses were observed at $2 \mathrm{~cm}$ and 3 $\mathrm{cm}$ using homogeneous tissue model. Clear $\mathrm{HHb}, \mathrm{tHB}$, and $\mathrm{rCCO}$ after regression seen in Fig. 6 indicate that the dynamics of changes measured at $3 \mathrm{~cm}$ and $4 \mathrm{~cm}$ channels was different from that measured at $1 \mathrm{~cm}$, and this difference can be attributed to the differences between the brain and scalp responses to BHs. Note that hemodynamic responses at $3 \mathrm{~cm}$ and $4 \mathrm{~cm}$ shown in Fig. 6 had significantly smaller magnitudes than those shown in Fig. 4 and Table 1. However, [rCCO] responses in both Fig. 4 and Fig. 6 had their magnitudes cloes to $0.1 \mu \mathrm{M}$. This result supports the conclusion that we succeeded in decoupling rCCO changes from changes in other chromophores, and that the measured rCCO changes occurred in the brain. We would like to underline that in both uniform and two- 
layer algorithms we used most conservative treatments of rCCO changes such as thresholding by the quantitative improvement in the goodness of fit.

The time courses of cerebral responses to BH in healthy adult humans were studied in detail using blood oxygen level dependent BOLD fMRI in [23, 24]. The quantitative relationship between the BOLD changes measured by fMRI and hemodynamic signals measured by NIRS was investigated in [32]. Fig 7 (adopted from [23]) shows averaged BOLD signal time courses with a $15 \mathrm{~s} \mathrm{BH}$ after expiration on areas supplied by the middle cerebral artery (MCA, red), anterior cerebral artery (ACA, blue) and posterior cerebral artery (PCA, black). Since our hNIRS probe was positioned on the left side of the forehead near the midline, it could interrogate cortical regions supplied by both ACA and MCA. The main difference between ACA and MCA regional responses was the early rise of the BOLD signal response in the ACA region and a $10 \mathrm{~s}$ delay in the MCA region. In our results the initial dynamics (during first $15 \mathrm{~s}$ of $\mathrm{BH}$ ) of cerebral $\mathrm{HbO} 2$ and $\mathrm{HHb}$ responses rather corresponded to the MCA type of the BOLD response [23,32]. A delayed rCCO response after the regression (Fig. 5) corresponds to the delayed ACA response. Such delayed cerebral responses were most different from the scalp hemodynamic responses to BHs measured at $1 \mathrm{~cm}$ (Fig. 3), which were also very significant.

Another significant difference between the long- and short-distance channels were longer peak times of hemodynamic responses in the short-distance channel (longer than $\mathrm{BH}$ duration Table 1). The peak times of responses to BHs after expiration measured by hNIRS at $4 \mathrm{~cm}$ were close to those measured by BOLD fMRI in [24] (see Fig. 2 in [24]).

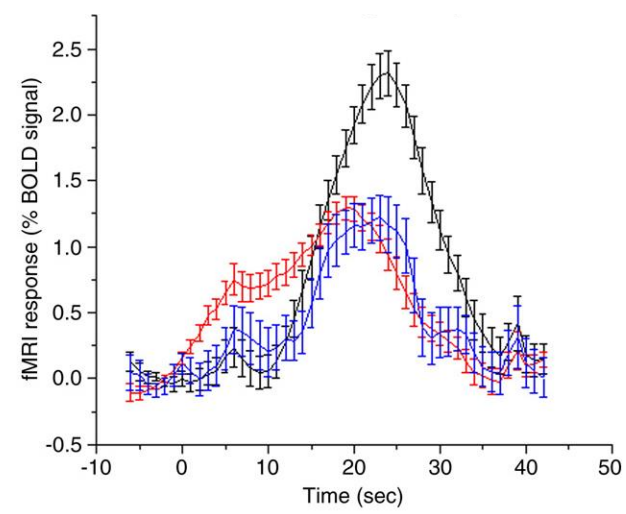

Figure 7. (Adopted from [23]) Averaged BOLD signal time course with breath holding of $15 \mathrm{~s}$ after expiration on areas supplied by the MCA (red), ACA (blue) and PCA (black).

Our results show that without using a short distance channel to remove biasing by scalp cerebral responses to BHs were most clear in [rCCO] time course at $4 \mathrm{~cm}$. Some features different from the scalp responses could also peep out in [HbO2] and [ $\mathrm{HHb}]$ time courses at $4 \mathrm{~cm}$. At $3 \mathrm{~cm}$ some features of cerebral response could be poorly recognized in the $[\mathrm{HHb}]$ time course. $[\mathrm{HbO} 2]$ time course did not show clear cerebral responses to $\mathrm{BH}$ neither at $\mathrm{cm}$ nor at $4 \mathrm{~cm}$, in particular die to the high inter-subject variability. However, measurement of [rCCO] changes required hyperspectral or many-wavelength multispectral technology.

We found the waveband $700-900 \mathrm{~nm}$ be optimal for the detection of cerebral responses to BHs in adults. The wavelength that exhibited the largest fractional change in the detected optical signal with respect to the baseline value both at 3 and $4 \mathrm{~cm}$ was between 800 and $850 \mathrm{~nm}$, which was in good agreement with the results of [13] (830 nm).

\section{Conclusions}

Cerebral changes could be efficiently separated from the extra-cerebral biasing using the time-domain linear regression of hNIRS signals measured at $3 \mathrm{~cm}$ and $4 \mathrm{~cm}$ sourcedetector separations by the signals from the short-distance channel. Without using the short-distance channel none of the signals measured at $3 \mathrm{~cm}$ clearly reflected cerebral 
changes. At $4 \mathrm{~cm}$ source-detector separation cerebral changes could be detected without using short-distance channels in the $[\mathrm{HHb}]$ and $[\mathrm{rCCO}]$ time courses. The clearest cerebral responses were detected in the $[\mathrm{rCCO}]$ time course at $4 \mathrm{~cm}$. The optimal waveband for the rCCO measurements was between $750 \mathrm{~nm}$ and $900 \mathrm{~nm}$. The [HbO2] time course at all source-detector distances was most dominated by the extracerebral biasing and therefore it was least suitable for the cerebral signal detection. Further clinical studies are required to investigate the ability of NIRS - breath holding paradigm to detect cerebral circulation disorders.

Author Contributions: “Conceptualization, S.L. and V.T.; methodology, V.T.; software, V.T.; validation, S.L., V.T. and Z.G.; formal analysis, Z.G.; investigation, Z.G.; resources, V.T.; data curation, V.T.; writing-original draft preparation, V.T.; writing-review and editing, S.L.; visualization, Z.G.; supervision, V.T.; project administration, V.T.; funding acquisition, V.T. All authors have read and agreed to the published version of the manuscript."

Funding: This research received no external funding.

Institutional Review Board Statement: The study was conducted according to the guidelines of the Declaration of Helsinki, and approved by the Research Ethics Board of Ryerson University (REB: 2008-003-1, May 4, 2015)."

Informed Consent Statement: Informed consent was obtained from all subjects involved in the study.

Data Availability Statement: In this section, please provide details regarding where data supporting reported results can be found, including links to publicly archived datasets analyzed or generated during the study. Please refer to suggested Data Availability Statements in section "MDPI Research Data Policies" at https: I www.mdpi.com l ethics. You might choose to exclude this statement if the study did not report any data.

Acknowledgments: “This research was supported by the Dean's Research Fund, Faculty of Science, Ryerson University".

Conflicts of Interest: The authors declare no conflict of interest.

\section{References}

1. F. Jöbsis, "Noninvasive, infrared monitoring of cerebral and myocardial oxygen sufficiency and circulatory parameters," Science, 198 (4323), 1264 -1267 (1977). http://dx.doi.org/10.1126/science.929199 SCIEAS 0036-8075

2. D. W.Green, and G. Kunst. "Cerebral oximetry and its role in adult cardiac, non-cardiac surgery and resuscitation from cardiac arrest." Anaesthesia 72, 48-57 (2017).

3. E. Okada, M. Firbank, M. Schweiger, S.R. Arridge, M. Cope, D.T. Delpy, "Theoretical and experimental investigation of nearinfrared light propagation in a model of the adult head." Applied optics 36 , 21-31 (1997).

4. I. Tachtsidis and F. Scholkmann, "False positives and false negatives in functional near-infrared spectroscopy: issues, challenges, and the way forward," Neurophotonics, 3 (3), 039801 (2016). https://doi.org/10.1117/1.NPh.3.3.039801 Google Scholar

5. Wyser, D., Mattille, M., Wolf, M., Lambercy, O., Scholkmann, F., \& Gassert, R. (2020). Short-channel regression in functional near-infrared spectroscopy is more effective when considering heterogeneous scalp hemodynamics. Neurophotonics, $7(3)$, 035011. https://doi.org/10.1117/1.NPh.7.3.035011

6. D. Milej, A. Abdalmalak, A. Rajaram, and K. St Lawrence. "Direct assessment of extracerebral signal contamination on optical measurements of cerebral blood flow, oxygenation, and metabolism." Neurophotonics 7, no. 4 (2020): 045002.

7. Fantini, Sergio, and Angelo Sassaroli. "Frequency-domain techniques for cerebral and functional near-infrared spectroscopy." Frontiers in neuroscience 14 (2020): 300

8. N. M. Gregg et al., "Brain specificity of diffuse optical imaging: improvements from superficial signal regression and tomography," Front. Neuroenerg., 214 (2010). https://doi.org/10.3389/fnene.2010.00014

9. L. Gagnon et al., "Improved recovery of the hemodynamic response in diffuse optical imaging using short optode separations and state-space modeling," NeuroImage, 56 (3), 1362 -1371 (2011). https://doi.org/10.1016/j.neuroimage.2011.03.001

10. M. A. Yücel et al., "Short separation regression improves statistical significance and better localizes the hemodynamic response obtained by near-infrared spectroscopy for tasks with differing autonomic responses," Neurophotonics, 2 (3), 035005 (2015). https://doi.org/10.1117/1.NPh.2.3.035005

11. R. B. Saager and A. J. Berger, "Direct characterization and removal of interfering absorption trends in two-layer turbid media," J. Opt. Soc. Am. A, 22 (9), 1874 -1882 (2005). https://doi.org/10.1364/JOSAA.22.001874 JOAOD6 0740-3232 
12. S. Brigadoi and R. J. Cooper, "How short is short? Optimum source-detector distance for short-separation channels in functional near-infrared spectroscopy," Neurophotonics, 2 (2), 025005 (2015). https://doi.org/10.1117/1.NPh.2.2.025005

13. Cheng X, Sie EJ, Boas DA, Marsili F. Choosing an optimal wavelength to detect brain activity in functional near-infrared spectroscopy. Opt Lett. 2021 Feb 15;46(4):924-927. doi: 10.1364/OL.418284. PMID: 33577549.

14. Dunne L, Hebden J, Tachtsidis I. Development of a near infrared multi-wavelength, multi-channel, time-resolved spectrometer for measuring brain tissue haemodynamics and metabolism. Adv Exp Med Biol. 2014;812:181-186. doi: 10.1007/978-1-4939-06208_24. Erratum in: Adv Exp Med Biol. 2014;812:E1. PMID: 24729231; PMCID: PMC4338597.

15. Song X, Chen X, Chen L, An X, Ming D. Performance Improvement for Detecting Brain Function Using fNIRS: A Multi-Distance Probe Configuration With PPL Method. Front Hum Neurosci. 2020 Nov 6;14:569508. doi: 10.3389/fnhum.2020.569508. PMID: 33240063; PMCID: PMC7677412.

16. Song X, Chen X, Wang Z, An X, Ming D. MBLL with weighted partial path length for multi-distance probe configuration of fNIRS. Annu Int Conf IEEE Eng Med Biol Soc. 2019 Jul;2019:4766-4769. doi: 10.1109/EMBC.2019.8857684. PMID: 31946927.

17. Giannoni L, Lange F, Tachtsidis I. Hyperspectral imaging solutions for brain tissue metabolic and hemodynamic monitoring: past, current and future developments. J Opt. 2018 Apr;20(4):044009. doi: 10.1088/2040-8986/aab3a6. Epub 2018 Mar 22. PMID: 29854375; PMCID: PMC5964611.

18. Chan ST, Ordway C, Calvanio RJ, Buonanno FS, Rosen BR, Kwong KK. Cerebrovascular Responses to O2-CO2 Exchange Ratio under Brief Breath-Hold Challenge in Patients with Chronic Mild Traumatic Brain Injury. J Neurotrauma. 2021 Jul 22. doi: 10.1089/neu.2021.0166. Epub ahead of print. PMID: 34210158.

19. McIntosh RC, Hoshi RA, Timpano KR. Take my breath away: Neural activation at breath-hold differentiates individuals with panic disorder from healthy controls. Respir Physiol Neurobiol. 2020 Jun;277:103427. doi: 10.1016/j.resp.2020.103427. Epub 2020 Feb 29. PMID: 32120012.

20. Lin W, Xiong L, Han J, Leung T, Leung H, Chen X, Wong KS. Hemodynamic effect of external counterpulsation is a different measure of impaired cerebral autoregulation from vasoreactivity to breath-holding. Eur J Neurol. 2014 Feb;21(2):326-31. doi: 10.1111/ene.12314. Epub 2013 Dec 7. PMID: 24313861.

21. I. Schelkanova and V. Toronov, "Independent component analysis of broadband near-infrared spectroscopy data acquired on adult human head," Biomed. Opt. Express 3, 64-74 (2012).

22. Lisa Holper M.D. and J. John Mann "Test-retest reliability of brain mitochondrial cytochrome-c-oxidase assessed by functional near-infrared spectroscopy," Journal of Biomedical Optics 23(5), 056006 (15 May 2018). https://doi.org/10.1117/1.JBO.23.5.056006

23. Leoni RF, Mazzeto-Betti KC, Andrade KC, de Araujo DB. Quantitative evaluation of hemodynamic response after hypercapnia among different brain territories by fMRI. Neuroimage. 2008 Jul 15;41(4):1192-8. doi: 10.1016/j.neuroimage.2008.03.035. Epub 2008 Apr 3. PMID: 18468457.

24. Pinto J, Bright MG, Bulte DP, Figueiredo P. Cerebrovascular Reactivity Mapping Without Gas Challenges: A Methodological Guide. Front Physiol. 2021 Jan 18;11:608475. doi: 10.3389/fphys.2020.608475. PMID: 33536935; PMCID: PMC7848198.

25. P. Shrout and J. Fleiss, "Intraclass correlations: uses in assessing rater reliability," Psychol. Bull., 86 (2), 420 -428 (1979). https://doi.org/10.1037/0033-2909.86.2.420 PSBUAI 0033-2909

26. Toronov V, Franceschini MA, Filiaci M, Fantini S, Wolf M, Michalos A, Gratton E. Near-infrared study of fluctuations in cerebral hemodynamics during rest and motor stimulation: temporal analysis and spatial mapping. Med Phys. 2000 Apr;27(4):801-15. doi: 10.1118/1.598943. PMID: 10798703.

27. Ghali MGZ, Ghali GZ. Mechanisms Contributing to the Generation of Mayer Waves. Front Neurosci. 2020 Jul 10;14:395. doi: 10.3389/fnins.2020.00395. PMID: 32765203; PMCID: PMC7381285.

28. Yeganeh, H., Toronov, V., Elliott, J., Diop, M., Lee, T., and St. Lawrence, K., "Broadband continuous-wave technique to measure baseline values and changes in the tissue chromophore concentrations", Biomedical Optics Express. 3(11), 2761 (2012).

29. Nosrati, R., Lin, S., Ramadeen, A., Monjazebi, D., Dorian, P., and Toronov, V., "Cerebral Hemodynamics and Metabolism During Cardiac Arrest and Cardiopulmonary Resuscitation Using Hyperspectral Near Infrared Spectroscopy", Circulation Journal. 81(6), 879-887 (2017).

30. F. Fabbri et al., “Optical measurements of absorption changes in two-layered diffusive media," Phys. Med. Biol. 49(7), 1183 (2004).

31. Baker WB, Parthasarathy AB, Ko TS, Busch DR, Abramson K, Tzeng SY, Mesquita RC, Durduran T, Greenberg JH, Kung DK, Yodh AG. Pressure modulation algorithm to separate cerebral hemodynamic signals from extracerebral artifacts. Neurophotonics. 2015 Jul;2(3):035004. doi: 10.1117/1.NPh.2.3.035004. Epub 2015 Aug 4. PMID: 26301255; PMCID: PMC4524732.

32. Toronov V, Walker S, Gupta R, Choi JH, Gratton E, Hueber D, Webb A. The roles of changes in deoxyhemoglobin concentration and regional cerebral blood volume in the fMRI BOLD signal. Neuroimage. 2003 Aug;19(4):1521-31. doi: 10.1016/s10538119(03)00152-6. PMID: 12948708. 